\title{
Orthodontic movement of a maxillary central incisor across the midline: a case report
}

\author{
Kiyong An, Dong-Soon Choi*, Insan Jang and Bong-Kuen Cha*
}

*Correspondence: dschoi@gwnu.ac.kr (or) korth@gwnu.ac.kr

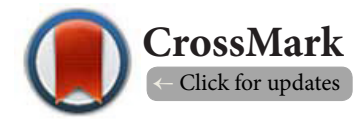

Department of Orthodontics, College of Dentistry, Gangneung-Wonju National University, Gangneung, South Korea.

\begin{abstract}
The maxillary central incisor can be lost due to dental injury, and it can be treated with orthodontic space closure. This case report presents the orthodontic treatment of a 22-year-old male patient who lost the maxillary right central incisor due to trauma. The maxillary left central incisor was moved across the midline to replace the missing right central incisor. The left lateral incisor was moved to replace the left central incisor, and the other maxillary left teeth were moved mesially to achieve a fully interdigitated Class II molar relationship. The left central and lateral incisors were reshaped as the right and left central incisors with porcelain crowns, and the final results were satisfactory in occlusion and esthetics. The effects of this alternative treatment on the midpalatal suture, nasopalatine foramen, labial frenum, and root apex of the central incisor are described.
\end{abstract}

Keywords: Incisor, injury, midpalatal suture, orthodontics, tooth movement

\section{Introduction}

A maxillary central incisor is the tooth most commonly affected by dental injury [1]. A missing maxillary central incisor can cause esthetic, psychological, and functional problems. The missing incisors need to be replaced with prosthesis such as implants. Orthodontic space closure [2] or autotransplantation with an immature premolar [3] can be alternative treatment plans. If the patient requires extraction of some teeth to relief crowding or to improve facial esthetics and occlusal function, the orthodontic space closure can be a better treatment approach than prosthetic replacement. Therefore, the position of missing tooth, occlusion, age, facial morphology, tooth morphology, and need for orthodontic treatment should be considered for the treatment plan.

The maxillary lateral incisor usually has been used as a substitute for a missing maxillary central incisor by orthodontic tooth movement $[\mathbf{2 , 4}]$. Infrequently, it is more favorable for an orthodontic strategy that the contralateral central incisor replaces the missing central incisor by moving across the midline. Cookson [5] presented a case report in which a maxillary right central incisor was moved across the midline to replace a left central incisor that was extracted due to a fusion. Follin [6] also presented a case report in which a left maxillary incisor was moved into the midline to close the space of right central incisor which was removed due to an odontoma. Other successful orthodontic movements of the maxillary central incisors to the contralateral side were reported in gemination cases [7,9], and dental trauma cases $[\mathbf{1 0}, \mathbf{1 1}]$.

The animal study wherein the central incisors were moved across the midline showed that the midpalatal suture was distorted in the direction of tooth movement in the young beagle dogs, whereas in the older beagle dogs whose sutures were already closed, the central incisors were moved across the midpalatal suture [12]. Most previously reported cases were initiated during the growth period in which the midpalatal sutures were patent $[\mathbf{6}, \mathbf{1 0}]$. In the recent report by Garib et al. [11], it was possible to move the maxillary central incisor through the midpalatal suture in a 19-year-old woman, but the midpalatal suture was also deviated to the same side of tooth movement. To date, there have been few case reports in which this alternative treatment was initiated in an adult patient. Therefore there is little information about the possibility of a tooth movement across the midpalatal suture.

This case report presents the orthodontic movement of a maxillary central incisor across the midline in an adult patient. In addition, the effects of this alternative treatment on the 
Choi et al. Oral Biology and Dentistry 2014,

midpalatal suture, nasopalatine foramen, labial frenum, and root apex of the central incisor are described.

\section{Diagnosis and etiology}

A 22-year-old man was referred to the Department of Orthodontics with the chief complaint of a missing maxillary right central incisor (Figure 1). Dental history showed a traumatic episode on the maxillary right central incisor one year ago. The patient showed a straight soft tissue profile and facial symmetry, but the nose deviated slightly to the left side. Intraoral examination showed a one-quarter cusp Class II molar relationship on the right side, a three-quarter cusp Class II molar relationship on the left side, and $2 \mathrm{~mm}$ of upper dental midline deviation to the left side. The maxillary left lateral incisor which was positioned palatally and overlapped with the left central incisor, showed crossbite with lower incisors. There was a moderate anterior crowding in the mandibular arch. The panoramic radiograph revealed a slight short root of the maxillary left central incisor. In the cephalometric analysis (Table 1), the patient had a mild Class II and vertical skeletal pattern (ANB $4.6^{\circ}$ and FMA $27.1^{\circ}$ ). The maxillary left central incisor was severely retroclined (U1 to $\mathrm{FH}, 90.5^{\circ}$ ), but the mandibular incisor was within normal limits (L1 to MP, $88.1^{\circ}$ ).

\section{Treatment objectives}

The treatment objectives were to close the space of the missing maxillary right central incisor, to align the maxillary and mandibular anterior teeth, and to achieve a good occlusion as a fully interdigitated Class II molar relationship on the left side by mesial movement of the left posterior teeth.

\section{Treatment alternatives}

Two different treatment alternatives were considered. The first option was to replace the maxillary right central incisor with an implant and extract the maxillary left lateral incisor (Figure 2A). The second treatment option was to close the space by moving the maxillary left central incisor across the midline and to move the left lateral incisor to the position of the left central incisor (Figure 2B). Both treatment options included moving mesially the maxillary left canine as a substitute for the left lateral incisor, and establishing a Class I molar relationship on the right side and a full Class II molar relationship on the left side. The options were proposed to the patient. Finally, the second alternative was chosen to avoid the implant and additional extraction of the natural tooth.

\section{Treatment progress}

MBT prescription self-ligating brackets (Clippy-C, 0.022- $\times 0.028$ -

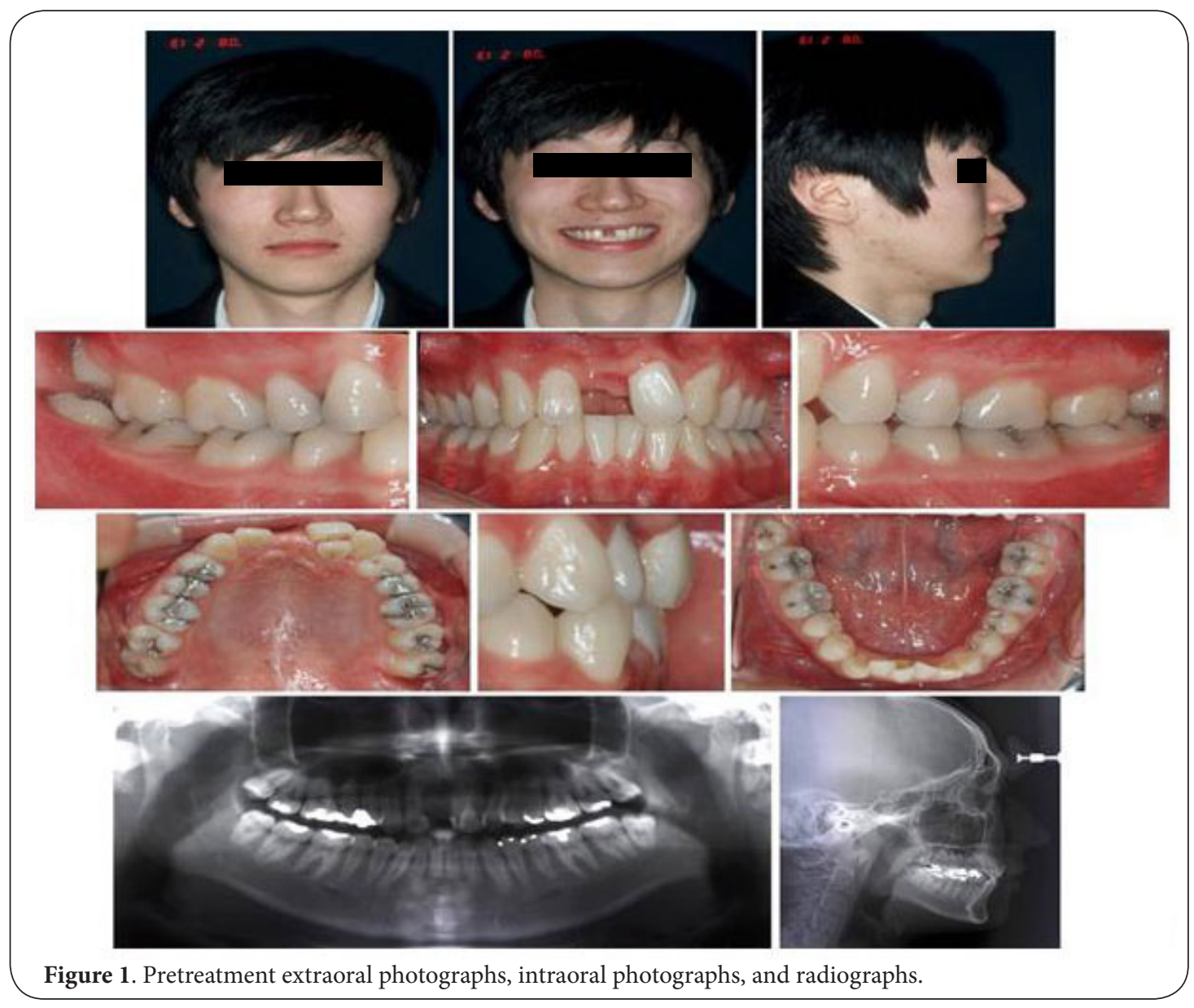


Choi et al. Oral Biology and Dentistry 2014,

http://www.hoajonline.com/journals/pdf/2053-5775-2-13.pdf

Table 1. Cephalometric measurements of pretreatment and posttreatment.

\begin{tabular}{lllll}
\hline Measurements & & Norm & Pre-treatment & Post-treatment \\
\hline SNA & $\left(^{\circ}\right)$ & 82.0 & 79.3 & 78.2 \\
SNB & $\left(^{\circ}\right)$ & 80.0 & 74.7 & 74.6 \\
ANB & $\left(^{\circ}\right)$ & 2.0 & 4.6 & 3.6 \\
Go-Me to FH & $\left(^{\circ}\right)$ & 22.7 & 27.1 & 27.0 \\
(FMA) & & & & \\
U1 to A-Pog & $(\mathrm{mm})$ & 6.2 & 1.2 & 6.7 \\
L1 to A-Pog & $(\mathrm{mm})$ & 3.0 & -1.5 & 2.4 \\
U1 to FH & $\left(^{\circ}\right)$ & 111.0 & 90.5 & 113.5 \\
L1 to MP & $\left(^{\circ}\right)$ & 90.0 & 88.1 & 96.9 \\
(IMPA) & & & & -3.2 \\
$\begin{array}{l}\text { Upper lip to } \\
\text { E-line }\end{array}$ & $(\mathrm{mm})$ & -4.8 & -4.5 & -1.3 \\
Lower lip to & $(\mathrm{mm})$ & -3.9 & -2.3 & \\
E-line & & & & \\
\hline
\end{tabular}

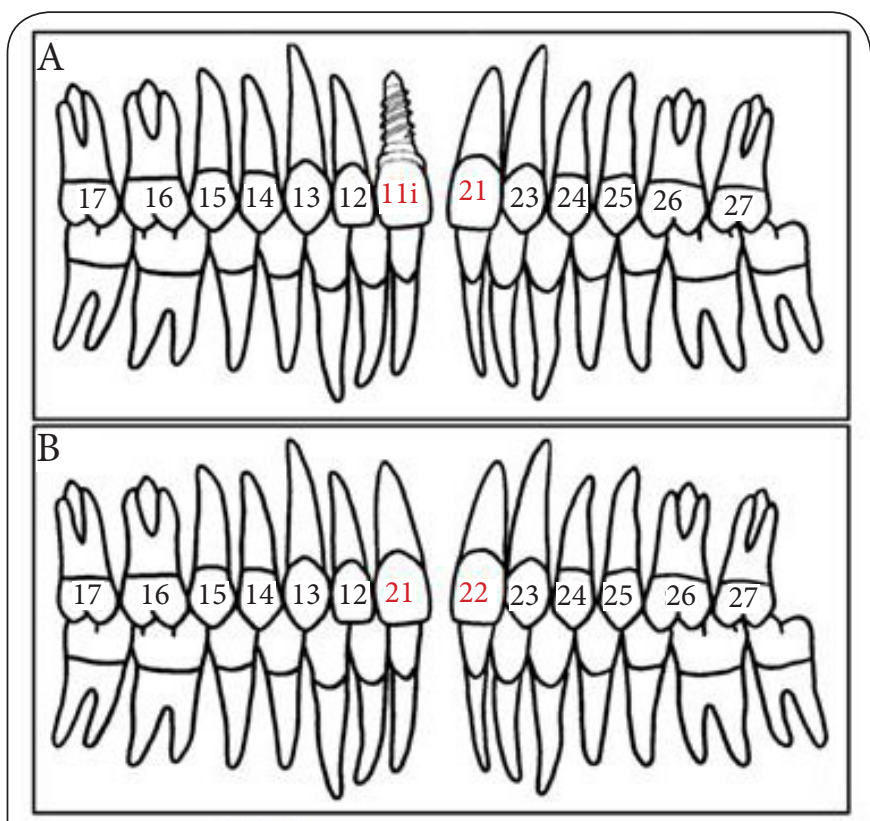

Figure 2. Treatment alternatives.

(A) The frist option was to replace the maxillary right central incisor with an implant and extract the maxillary left lateral incisor.

(B) The second option was to close the missing space by moving the maxillary left central incisor across the midline and to move the left lateral incisor to the position of left central incisor.

inch slot, Tomy, Japan) were bonded in the maxillary teeth. Leveling and alignment were initiated with 0.014 -inch nickeltitanium (NiTi) arch wire (Figure 3A), followed by 0.018 -inch stainless-steel (SS) arch wire. The left central incisor was moved to the right side across the midline using the compressed open coil spring (Figure 3B).

To establish a full Class II molar relationship on the left side, the left posterior teeth were mesially moved with the miniscrew placed between the first and second premolars (Figure 3C). After nine months of treatment, the left central incisor was successfully moved to the contralateral side, then, the maxillary left lateral incisor was labially moved using 0.014 -inch $\mathrm{NiTi}$ arch wire overlayed onto the 0.018 -inch SS arch wire (Figure 3D), followed by $0.019-\times 0.025$-inch beta-titanium arch wire with L-loops for the torque control of the left lateral incisor.

After 18 months from the beginning of treatment, the mandibular teeth were bonded with brackets and aligned using $0.016-, 0.016-\times 0.022-$, and $0.019-\times 0.025$-inch NiTi arch wire for 12 months. After completion of leveling and alignment in the maxillary and mandibular arch, correction of the dental midline and molar relationship was accomplished with both miniscrews anchorage and a Class III interarch elastic on the left side (Figure 3E). The maxillary right posterior teeth were slightly distalized, and the maxillary left posterior teeth were mesialized. The duration of treatment was three years and six months.

After removal of the fixed orthodontic appliances, the mesially moved maxillary left canine was recontoured for esthetics. The patient was referred to the Department of Prothodontics to reshape the left central and lateral incisor as the right and left central incisors with porcelain veneer crowns. Maxillary and mandibular retention was performed with bonded canine-tocanine fixed retainers.

\section{Results}

The soft tissue profile remained facial harmony with minimally changed lips (Figure 4). The maxillary right central incisor was successfully replaced with the left central incisor. An ideal overjet, overbite, coincident dental midlines, and better interdigitations of the posterior teeth were achieved.

In cephalometric superimposition, the retroclined maxillary central incisor was protruded in ideal position and inclination (Figure 5 and Table 1). In evaluation of the three-dimensional digital models superimposed using the palatal surface as reference area [13], the maxillary left central incisor was moved $9 \mathrm{~mm}$ to the right side across the midline and tipped labially $5 \mathrm{~mm}$ (Figure 6). The maxillary left lateral incisor showed $1.5 \mathrm{~mm}$ of medial and $8 \mathrm{~mm}$ of labial movement.

In the comparison of serial periapical radiographs, however, the root resorption of the maxillary left central incisor developed during the treatment (Figure 7). In the cone-beam computed tomography (CBCT) images taken at five months after removal of the orthodontic appliances, the midpalatal suture was observed to be deviated to the right side (Figures $\mathbf{8 A}$ and $\mathbf{8 B}$ ). The incisive foramen was also distorted in the same direction of tooth movement (Figure 8E).

The extraoral and intraoral photographs at five months of postretention showed a stable occlusion and esthetic smile by orthodontic and prosthetic treatment without any relapse (Figure 9). 
Choi et al. Oral Biology and Dentistry 2014,

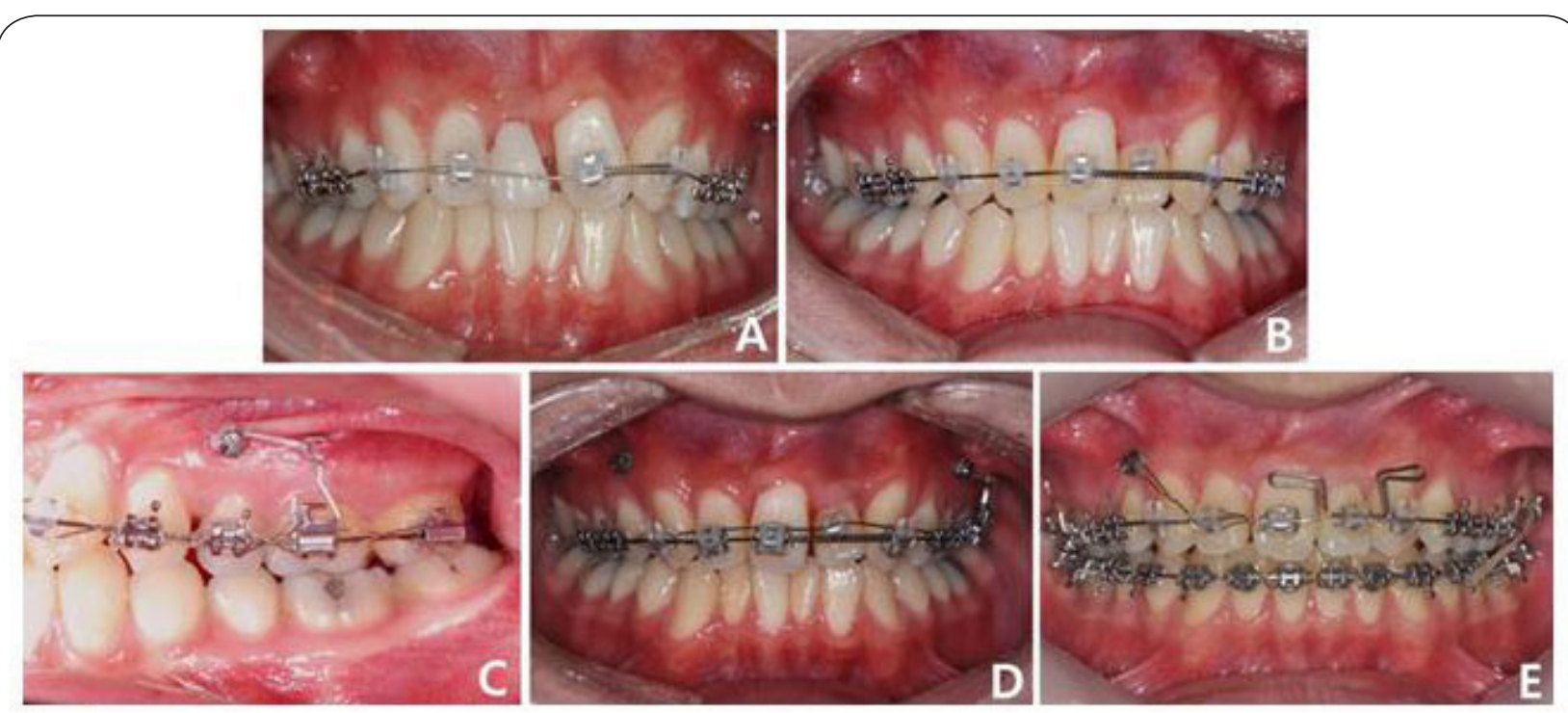

Figure 3. Treatment progress.

(A) Leveling using 0.014-inch NiTi arch wire.

(B) Movement of the left central incisor to the right side across the midline using the open coil spring on 0.018-inch SS arch wire.

(C) Mesial movement of the left posterior teeth using the miniscrew as an anchorage.

(D) Labial movement of the left lateral incisor using overlayed 0.014-inch NiTi arch wire. The miniscrews in the right and left side were used for the distalization and mesialization of the maxillary posterior teeth, respectively.

(E) Correction of the dental midline and molar relationship. L-loops were used for the torque control of the left lateral incisor.

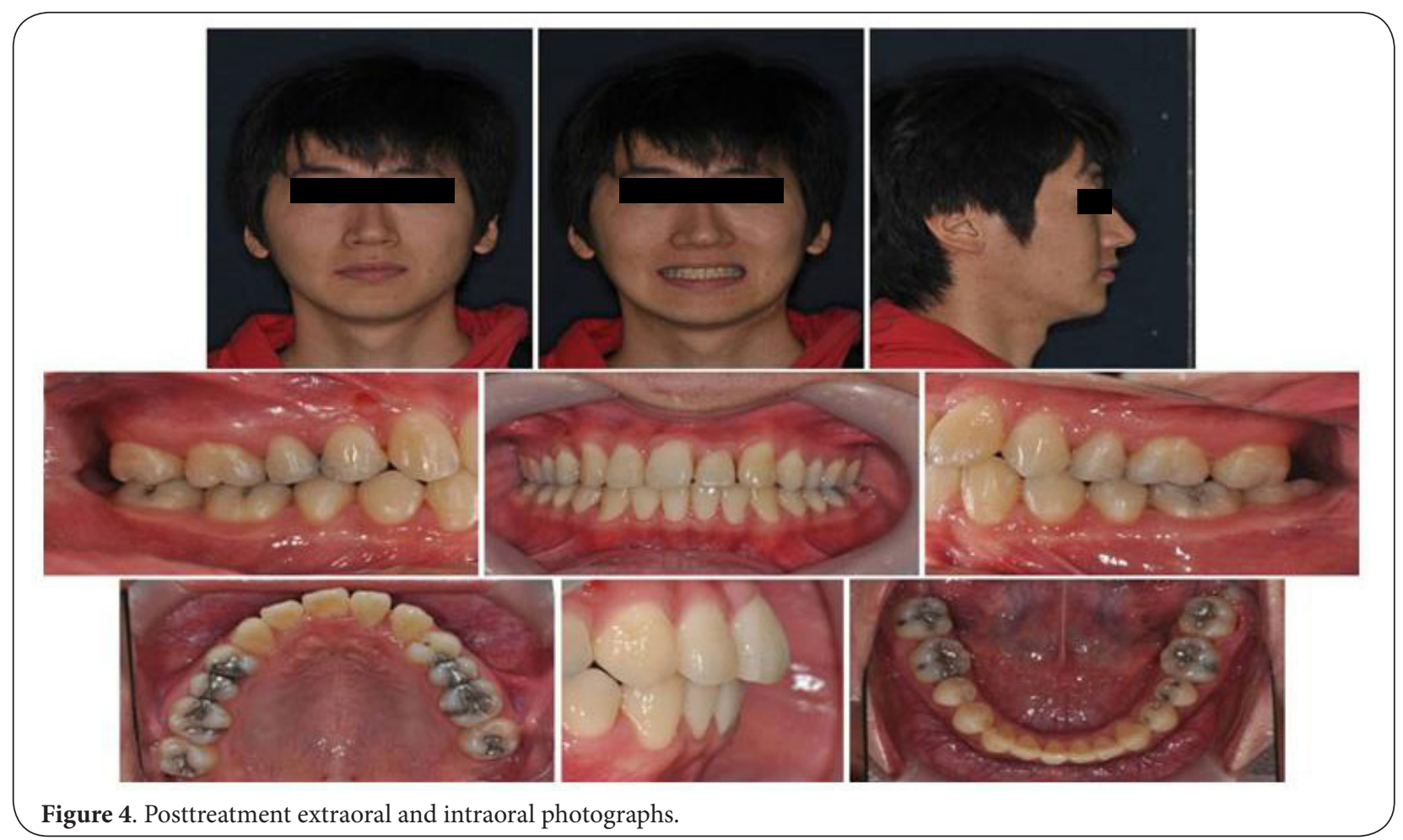

\section{Discussion}

In most previous case reports $[\mathbf{6 , 1 0}]$, the patients were treated during the growth period in which the midpalatal sutures were patent. They showed that the central incisors, which moved across the midline, did not pass through the midpalatal suture, but the suture was distorted in the same direction of tooth movement. 

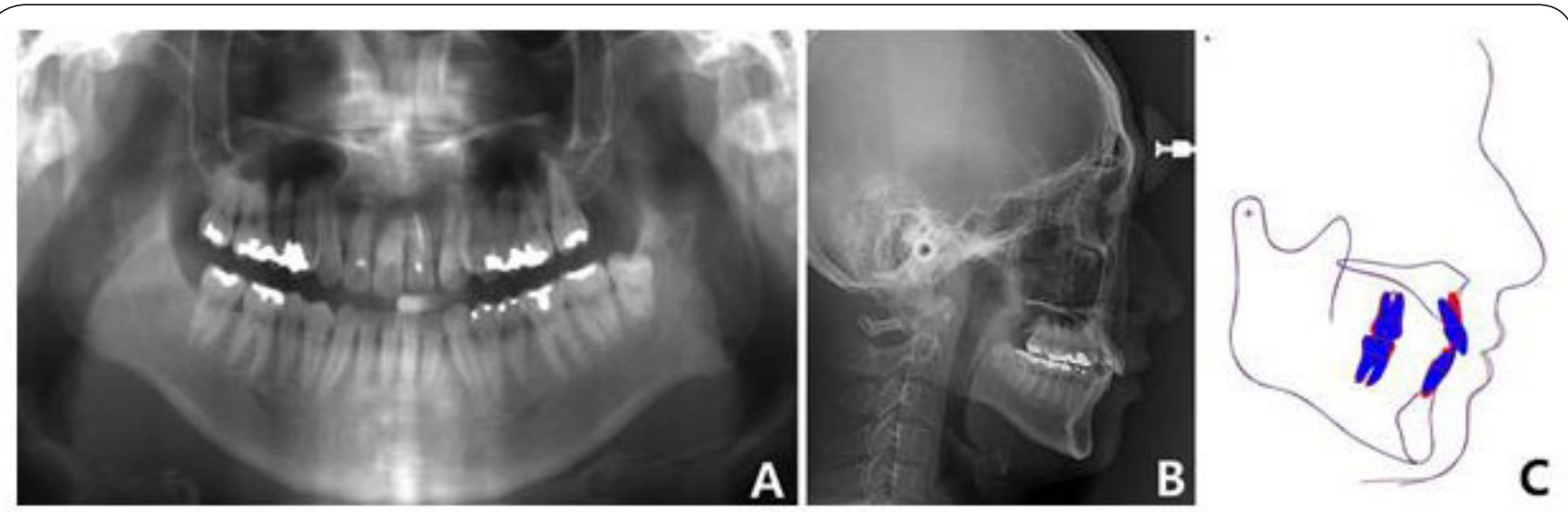

Figure 5. (A) Posttreatment panoramic radiograph.

(B) Cephalometric radiographs.

(C) Superimposition of the pretreatment (red) and posttreatment (blue) cephalometric tracings.

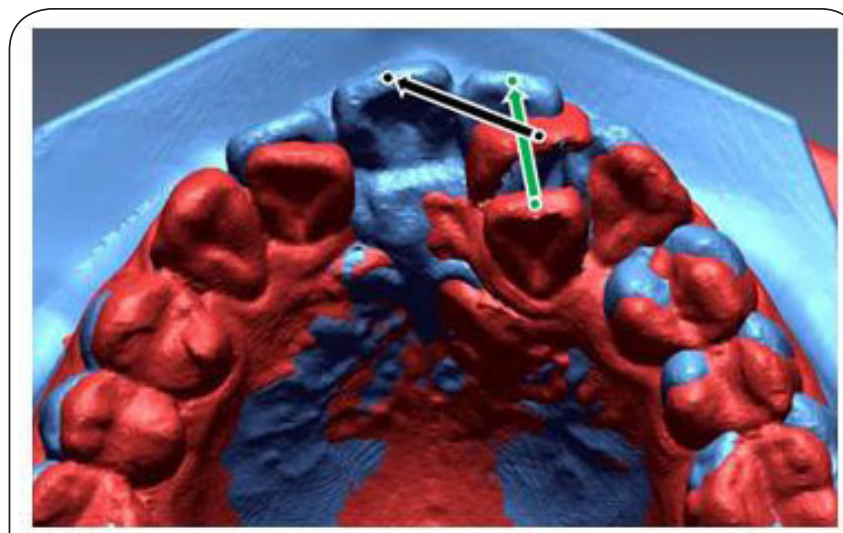

Figure 6. Superimposition of the pretreatment (red) and posttreatment (blue) 3D digital models. The maxillary left central incisor was moved $9 \mathrm{~mm}$ to the right side and tipped labially $5 \mathrm{~mm}$ (black arrow). The left lateral incisor showed 1.5 $\mathrm{mm}$ of medial and $8 \mathrm{~mm}$ of forward movement (green arrow).

These results were in agreement with the results in the young beagles by Follin et al [12]. On the other hand, they reported that in the old beagles the central incisors moved across the midpalatal suture without any impediment. The present case was performed in an adult patient whose palatal suture was expected to be closed, and the maxillary left central incisor was successfully moved to the position of the right central incisor. In the CBCT image, however, the central incisor did not pass across the midpalatal suture (Figure $\mathbf{8 B}$ ). The reason may be that as shown in the CBCT image (Figure $\mathbf{8 A}$ ), the midpalatal suture was still open although the patient was 26 years old. The midpalatal suture from birth to adulthood is known to become wavy and interdigitated with aging [14]. According to Persson and Thilander [15], marked closure is hardly observed until the third decade of life. Consequently, it remains a question whether the central incisor can move across the closed midpalatal suture in humans.
The tooth movement across the midline did not affect the nasopalatine canal (Figures $\mathbf{8 C}$ and $\mathbf{8 D}$ ), and it was in accordance with the findings of Bosio et al [10]. However, the incisive foramen, the palatal orifice of the nasopalatine canal for the nasopalatine nerve and artery, was distorted in the direction of tooth movement (Figure 8E). The soft tissue tension associated with the incisor movement across the midline might change the incisive foramen by bone remodeling process. Although there were no clinical symptoms in the present case, it may need to carefully consider the spatial position between the maxillary central incisor and the incisive foramen during this treatment.

In the comparison of Figures 7A and 7B, no obvious sign of root resorption was detected during the initial movement of the left central incisor across the midline, but significant root resorption was observed after removal of orthodontic appliances (Figures 7D and 7E). Follin et al., [12] showed root resorption of the cementum and dentin in old animals with closed midpalatal suture. However, the midpalatal suture of the present case was not closed. The other factors for root resorption may be the dental trauma prior to orthodontic treatment. Brin et al., [16] reported that the prevalence of root resorption was higher in orthodontic patients with traumatized teeth than in orthodontic patients with intact incisors and control group. Also, it has been reported that abnormal root shape such as blunted, short, pipette, or dilacerated shape, as well as long duration of treatment were related with higher risk of root resorption $[17,18]$. The patient in the present case had a history of dental trauma on the maxillary incisor region, and the root of left central incisor and lateral incisor showed short and dilacerated shape at the pretreatment radiography. In addition, the duration of treatment was too long, because the leveling in the mandibular arch was initiated after correction of the crossbite of the lateral incisor.

The frenum and gingival tissue tend to move in the direction of tooth movement [19]. The remodeling of the fibrils between maxillary frenum attachments and the alveolar process need a very long period [20]. To prevent the reopening of the space, 
Choi et al. Oral Biology and Dentistry 2014,

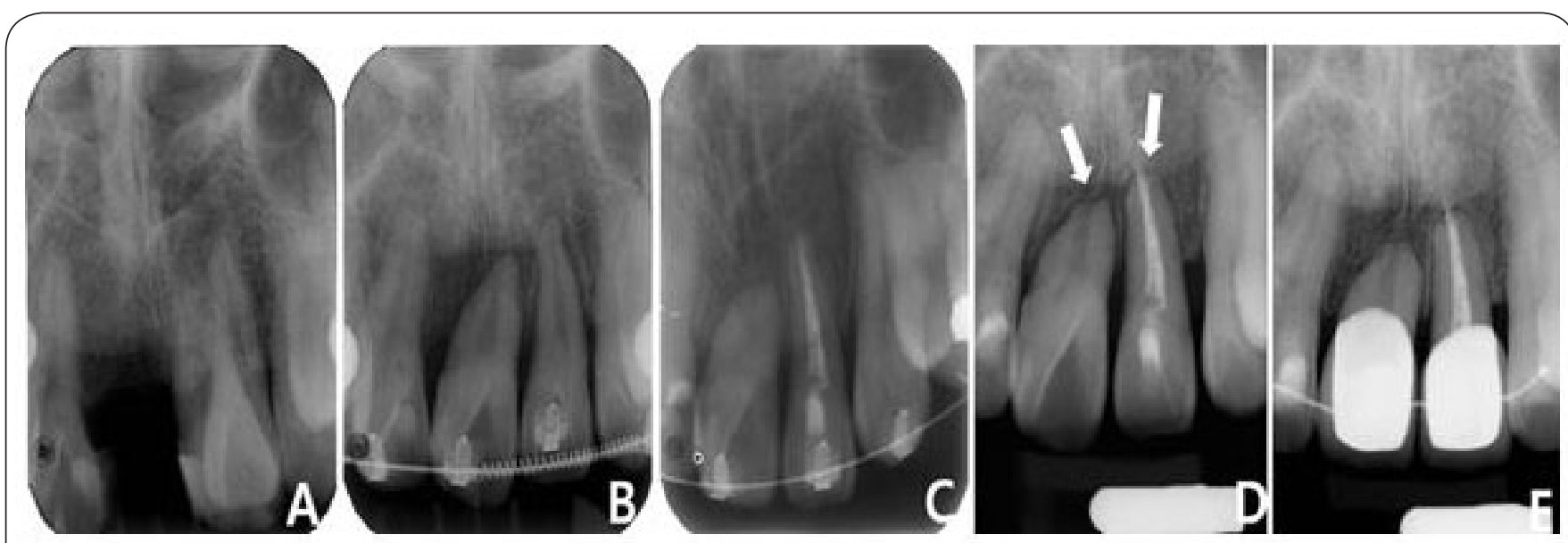

Figure 7. Serial periapical radiographs.

(A) Pretreatment.

(B) Nine months of treatment.

(C) 29 months of treatment. The lateral incisor was endodontically treated due to a hypersensitivity.

(D) At the time of appliance removal. The left central and lateral incisor showed an external root resorption (arrows).

(E) At five months of postretention. The root resorption did not progressed.

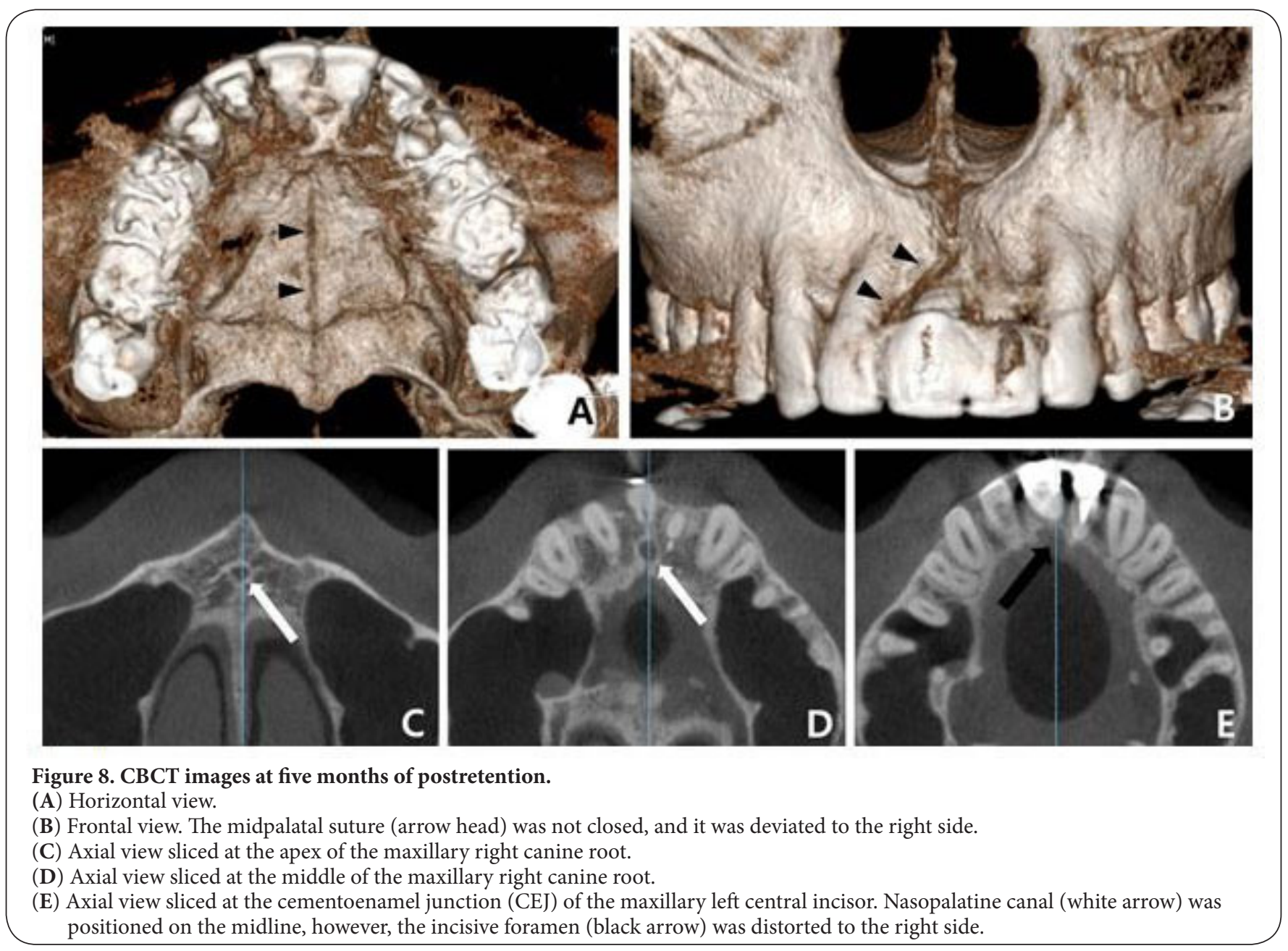




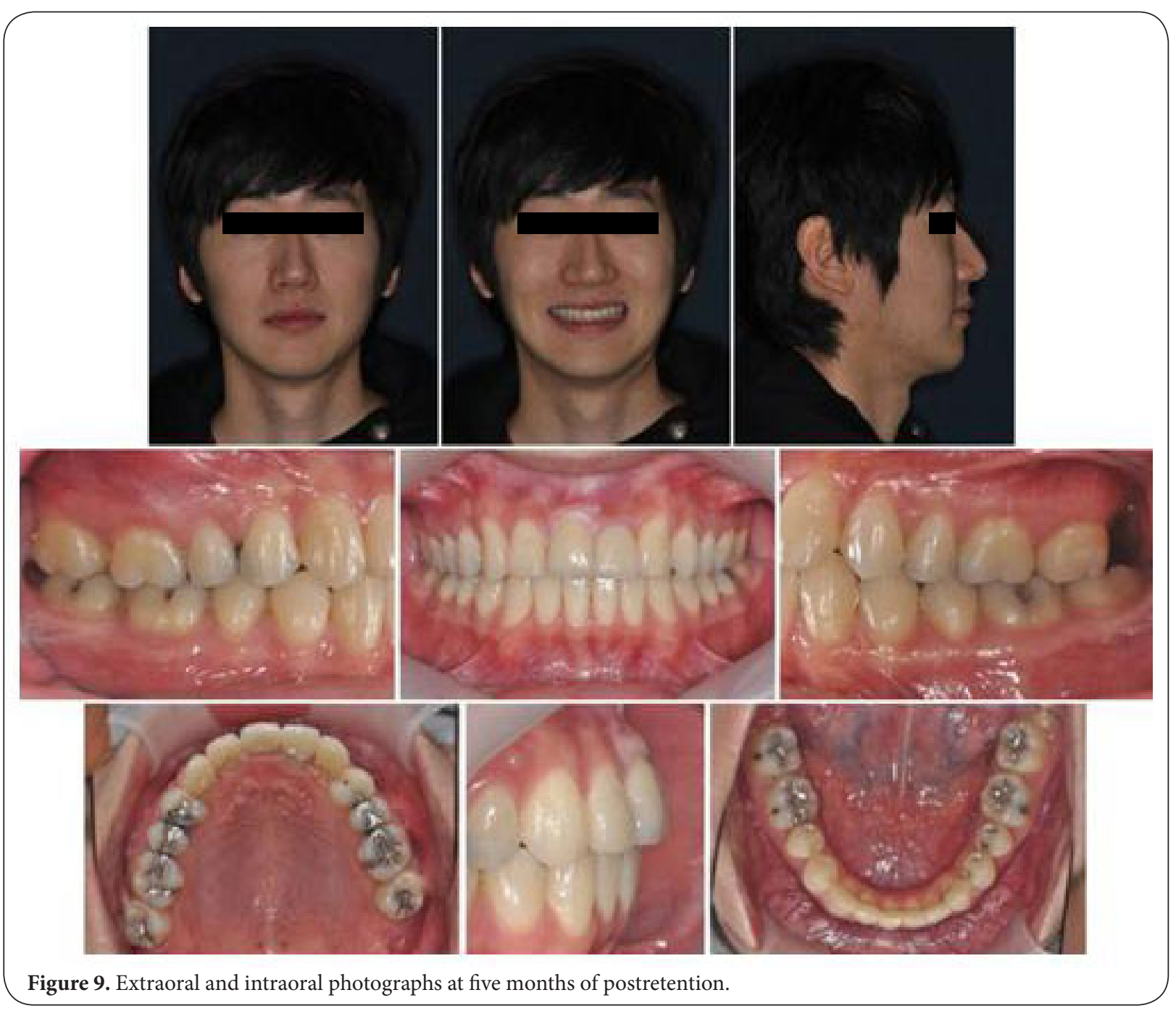

fixed lingual retainer or frenectomy were recommended by many authors [7,9-11]. In the present case, the fixed lingual retainer was used without frenectomy, because the patient showed that the insertion of frenum was far from the alveolar ridge.

There was no bleeding on probing, even though a generalized alveolar bone resorption were observed around the anterior teeth from the initiation of treatment (Figure 7A). The irreversible periodontal changes such as gingival recession and the progressive alveolar bone loss did not occur during the orthodontic treatment and the retention period. The fixed lingual retainer may protect the anterior teeth from the occlusal force which would be a traumatic force to the periodontally destroyed teeth.

Some clinical procedures may improve the orthodontic results in cases of missing maxillary incisors. It includes: (1) mesiodistal positioning and angulation of mesially moved lateral incisor, (2) intrusion of the lateral incisor and extrusion of the canine to achieve optimal alveolar bone height and gingival contours, (3) recontouring of the canine cusp, (4) mesial rotation of the first premolar, (5) and gingivectomy on the lateral incisor to increase clinical crown height $[\mathbf{2}, \mathbf{2 1}]$. In the present case, the inclination of the left lateral incisor was not fully corrected due to apical root resorption. The maxillary left first premolar was not mesially rotated because there was no occlusal interference between the palatal cusp of the first premolar and the lower canine. The gingivectomy on the left lateral incisor for esthetics was refused by the patient, but the patient was pleased with the final results in esthetics and occlusal function.

\section{Conclusion}

It was possible to move the maxillary central incisor across the midline in the adult patient, however, the central incisor did not move across the midpalatal suture. The midpalatal suture, incisive foramen, incisive papilla, and labial frenum were deviated to the direction of tooth movement.

\section{Competing interests}

The authors declare that they have no competing interests.

\section{Authors' contributions}

All authors contributed equally to the manuscript. 
Choi et al. Oral Biology and Dentistry 2014,

http://www.hoajonline.com/journals/pdf/2053-5775-2-13.pdf

doi: $10.7243 / 2053-5775-2-13$

\section{Publication history}

Received: 14 October 2014 Revised: 17 November 2014

Accepted: 01 December 2014 Published: 08 December 2014

\section{References}

1. Grimm, S., Frazao, P., Antunes, J. L., Castellanos, R. A. and Narvai, P. C. (2004). Dental injury among Brazilian schoolchildren in the state of Sao Paulo. Dent Traumatol, 20, 134-8.

2. Zachrisson, B. U. (1978). Improving orthodontic results in cases with maxillary incisors missing. Am J Orthod, 73, 274-89.

3. Mendoza Mendoza, A., Solano Reina, E. and Segura-Egea, J. J. (2010). Treatment of an avulsed maxillary permanent central incisor replaced by autotransplantation of a mandibular premolar: 14-year follow-up. Int Endod J, 43, 818-27.

4. Czochrowska, E. M., Skaare, A. B., Stenvik, A. and Zachrisson, B. U. (2003). Outcome of orthodontic space closure with a missing maxillary central incisor. Am J Orthod Dentofacial Orthop, 123, 597-603.

5. Cookson, A. M. (1981). Movement of an upper central incisor across the midline. Br J Orthod, 8, 59-60.

6. Follin, M. E. (1985). Orthodontic movement of maxillary incisor into the midline. A case report. Swed Dent J, 9, 9-13.

7. Melnik, A. K. (1993). Orthodontic movement of a supplemental maxillary incisor through the midpalatal suture area. Am J Orthod Dentofacial Orthop, 104, 85-90.

8. McCollum, A. G. (1999). Crossing the midline: a long-term case report. Am J Orthod Dentofacial Orthop, 115, 559-62.

9. Pair, J. (2011). Movement of a maxillary central incisor across the midline. Angle Orthod, 81, 341-9.

10. Bosio, J. A., Bradley, T. G. and Hefti, A. F. (2011). Moving an incisor across the midline: a treatment alternative in an adolescent patient. Am J Orthod Dentofacial Orthop, 139, 533-43.

11. Garib, D. G., Janson, G., dos Santos, P. B., de Oliveira Baldo, T., de Oliveira, G. U. and Ishikiriama, S. K. (2012). Orthodontic movement of a maxillary incisor through the midpalatal suture: a case report. Angle Orthod, 82, 370-9.

12. Follin, M., Ericsson, I. and Thilander, B. (1984). Orthodontic movement of maxillary incisors through the midpalatal suture area--an experimental study in dogs. Eur J Orthod, 6, 237-46.

13. Cha, B. K., Lee, J. Y., Jost-Brinkmann, P. G. and Yoshida, N. (2007). Analysis of tooth movement in extraction cases using three-dimensional reverse engineering technology. Eur J Orthod, 29, 325-31.

14. Melsen, B. (1975). Palatal growth studied on human autopsy material. A histologic microradiographic study. Am J Orthod, 68, 42-54.

15. Persson, M. and Thilander, B. (1977). Palatal suture closure in man from 15 to 35 years of age. Am J Orthod, 72, 42-52.

16. Brin, I., Ben-Bassat, Y., Heling, I. and Engelberg, A. (1991). The influence of orthodontic treatment on previously traumatized permanent incisors. Eur J Orthod, 13, 372-7.

17.Nigul, K. and Jagomagi, T. (2006). Factors related to apical root resorption of maxillary incisors in orthodontic patients. Stomatologija, 8, 76-9.

18. Sameshima, G. T. and Sinclair, P. M. (2004). Characteristics of patients with severe root resorption. Orthod Craniofac Res, 7 , 108-14.

19. McCollum, A. G. and Preston, C. B. (1980). Maxillary canine retraction, periodontal surgery, and relapse. Am J Orthod, 78,
610-22.

20. Thilander, B. (2000). Biological basis for orthodontic relapse. Semin Orthod, 6, 195-205.

21. Romano, R., Bichacho, N. and Touati, B. (2005). The art of the smile: integrating prosthodontics, orthodontics, periodontics, dental technology, and plastic surgery in esthetic dental treatment. Quintessence Pub.

\section{Citation:}

An K, Choi DS, Jang I and Cha BK. Orthodontic movement of a maxillary central incisor across the midline: a case report. Oral Biol Dent. 2014; 2:13. http://dx.doi.org/10.7243/2053-5775-2-13 